\title{
Cardiac xenotransplantation technology provides materials for improved bioprosthetic heart valves
}

\author{
Christopher G. A. McGregor, MB, FRCS, MD(Hons), ${ }^{\text {a,b }}$ Alain Carpentier, MD, $\mathrm{PhD},{ }^{\mathrm{c}}$ \\ Nermine Lila, DVM, PhD, ${ }^{\mathrm{c}}$ John S. Logan, PhD, ${ }^{\mathrm{d}}$ and Guerard W. Byrne, PhD ${ }^{\mathrm{a}, \mathrm{b}}$
}

\begin{abstract}
Objectives: Human subjects and Old World primates have high levels of antibody to galactose- $\alpha-1,3$ galactose $\beta-1,4-\mathrm{N}$-acetylglucosamine ( $\alpha$-Gal). Commercially available bioprosthetic heart valves of porcine and bovine origin retain the $\mathrm{Gal}$ antigen despite current processing techniques. Gal-deficient pigs eliminate this xenoantigen. This study tests whether binding of human anti-Gal antibody effects calcification of wild-type and Galdeficient glutaraldehyde-fixed porcine pericardium by using a standard subcutaneous implant model.
\end{abstract}

Methods: Expression of $\alpha$-Gal was characterized by lectin Griffonia simplicifolia-IB4 staining. Glutaraldehyde-fixed pericardial disks from Gal-positive and Gal-deficient pigs were implanted into 12-dayold Wistar rats and 1.5-kg rabbits with and without prelabeling with affinity-purified human anti-Gal antibody. Calcification of the implants was determined after 3 weeks by using inductively coupled plasma spectroscopy.

Results: The $\alpha$-Gal antigen was detected in wild-type but not Gal-deficient porcine pericardium. Wild-type disks prelabeled with human anti-Gal antibody exhibited significantly greater calcification compared with that seen in antibody-free wild-type samples (mean \pm standard error of the mean: $111 \pm 8.4$ and $74 \pm 9.6 \mathrm{mg} / \mathrm{g}$, respectively; $P=.01$ ). In the presence of anti-Gal antibody, a significantly greater level of calcification was detected in wild-type compared with GTKO porcine pericardium $(111 \pm 8.4$ and $55 \pm 11.8 \mathrm{mg} / \mathrm{g}$, respectively; $P=.005)$. Calcification of Gal-deficient pericardium was not affected by the presence of anti-Gal antibody $(51 \pm 9.1$ and $55 \pm 11.8 \mathrm{mg} / \mathrm{g})$.

Conclusions: In this model anti-Gal antibody accelerates calcification of wild-type but not Gal-deficient glutaraldehyde-fixed pericardium. This study suggests that preformed anti-Gal antibody present in all patients might contribute to calcification of currently used bioprosthetic heart valves. Gal-deficient pigs might become the preferred source for new, potentially calcium-resistant bioprosthetic heart valves. (J Thorac Cardiovasc Surg 2011;141:269-75)

Survival of pig-to-primate cardiac xenotransplantation has improved through greater understanding of the mechanism of rejection and the development of new technologies to block xenograft rejection. ${ }^{1}$ Rejection of vascularized xenografts is dominated by antibody-mediated processes, including complement-mediated injury and endothelial cell activation, which results in xenograft rejection characterized by widespread microvascular thrombosis and coagulative necrosis. One key antigen in this process is ga-

\footnotetext{
From the Department of Cardiothoracic Surgery, ${ }^{\text {a }}$ University College London, London, United Kingdom; the Division of Cardiovascular Surgery, ${ }^{\mathrm{b}}$ Mayo Clinic, Rochester, Minn; the Department of Cardiac Surgery, ${ }^{\mathrm{c}}$ University of Paris, Paris, France; and FIOS Therapeutics, Inc, ${ }^{\mathrm{d}}$ Rochester, Minn.

Supported by the Mayo Clinic and the University of Paris Descartes, Assistance Publique Hôpitaux de Paris, and the Alain Carpentier Foundation.

Disclosures: Drs McGregor and Logan are inventors of technology related to xenotransplantation that has been licensed by the Mayo Clinic to a commercial entity.

Read at the 90th Annual Meeting of The American Association for Thoracic Surgery, Toronto, Ontario, Canada, May 1-5, 2010.

Received for publication June 21, 2010; revisions received Aug 11, 2010; accepted for publication Aug 23, 2010.

Address for reprints: Christopher G. A. McGregor, MB, FRCS, MD (Hons), Mayo Clinic, 9-255 Charlton Building, 200 First Str SW, Rochester, MN 55905 (E-mail: mcgregor.christopher@mayo.edu).

$0022-5223 / \$ 36.00$

Copyright (c) 2011 by The American Association for Thoracic Surgery doi:10.1016/j.jtcvs.2010.08.064
}

lactose- $\alpha$-1,3-galactose $\beta-1,4-\mathrm{N}$-acetylglucosamine $\quad(\alpha$ $\mathrm{Gal})$, which has been identified as the predominant xenograft rejection antigen present in the wild-type pig. ${ }^{2}$ All mammalian species except human subjects and Old World primates produce the $\alpha$-Gal antigen. ${ }^{3}$ In pigs the $\alpha$-Gal antigen is present on glycoproteins and glycolipids, is expressed at high levels by vascular endothelial cells, and might be present at up to $10^{11}$ epitopes per milligram of tissue matrix. ${ }^{4}$ Human subjects and Old World primates produce anti-Gal antibody, which could be up to $1 \%$ of circulating immunoglobulin (Ig) $\mathrm{G}$ and arises early in life in response to stimulation by intestinal microflora. ${ }^{5}$

Methods have been developed to block or remove antiGal antibody to prolong vascular xenograft survival ${ }^{6,7}$; however, the advent of somatic cell nuclear transfer made it possible to use homologous recombination in cultured fibroblasts to disrupt the $\alpha-1,3$ galactosyltransferase (GGTA1) gene and thereby derive mutant pigs deficient in the synthesis of the $\alpha$-Gal antigen (GTKO pigs). ${ }^{8,9}$ These GTKO pigs do not express the $\alpha$-Gal antigen, and vascular pig-to-primate xenografts with GTKO organs do not induce an anti-Gal immune response. ${ }^{10}$ Because this major xenogeneic antigen is eliminated from these animals, they might be a useful source of material for the development of new bioprosthetic heart valves (BHVs). 


$$
\begin{aligned}
& \text { Abbreviations and Acronyms } \\
& \begin{aligned}
\alpha-\mathrm{Gal} & =\text { galactose- } \alpha-1,3 \text { galactose } \beta-1,4-\mathrm{N}- \\
& \text { acetylglucosamine } \\
\text { BHV } & =\text { bioprosthetic heart valve } \\
G G T A 1 & =\alpha-1,3 \text { galactosyltransferase } \\
\text { GSIB } 4 & =\text { Griffonia simplicifolia-IB4 } \\
\text { Ig } & =\text { immunoglobulin } \\
\text { LVAD } & =\text { left ventricular assist device } \\
\text { PBS } & =\text { phosphate-buffered saline }
\end{aligned}
\end{aligned}
$$

BHVs are commonly produced from glutaraldehyde-fixed porcine aortic valves or sewn from fixed bovine pericardium. Such valves generally do not require anticoagulation and have a low level of thrombogenicity. BHVs are used principally in older patients because they are more durable in this population and because BHV degeneration occurs more rapidly in younger patients. BHV degeneration, which involves extensive calcification, has been attributed to excess glutaraldehyde, mechanical failure, collagen degradation, and calcium precipitation by residual lipids. There might also be an immunologic mechanism or mechanisms that lead to valve degeneration because fixation methods do not completely eliminate device antigenicity ${ }^{11,12}$ and patients implanted with BHVs exhibit increased levels of anti-Gal reactive antibody. ${ }^{13}$ In this study we investigate the potential for anti-Gal antibody to promote calcification using the subcutaneous implant model in rats and rabbits. We compare the level of calcification of wild-type and GTKO glutaraldehyde-fixed pericardium with and without exposure to human anti-Gal antibody.

\section{MATERIALS AND METHODS \\ Anti-Gal Antibody}

Human anti-Gal antibody was affinity purified from human AB plasma with $\alpha$-Gal Sepharose columns (Nextran, Princeton, NJ). ${ }^{14}$ Antibody was recovered from the column by using free $\alpha$-Gal trisaccharide competition and was dialyzed before storage at $-80^{\circ} \mathrm{C}$. For labeling-fixed porcine pericardial disks, anti-Gal antibody $(4 \mu \mathrm{g} / \mathrm{mL})$ was diluted into phosphatebuffered saline (PBS) and sterile filtered.

\section{Calcium Determination}

Calcium levels were determined by the Mayo Clinic trace metals laboratory, Department of Laboratory Medicine and Pathology. In brief, dried pericardium samples were weighed and extracted at $76^{\circ} \mathrm{C}$ with concentrated nitric acid and $30 \%$ hydrogen peroxide. Samples were diluted in $1 \% \mathrm{HCl}$ and loaded into an Optimas Inductively Coupled Plasma ICP emission instrument. Calcium was measured at $317.9 \mathrm{~nm}$ and yttrium at $371.0 \mathrm{~nm}$ as an internal control. A standard curve for calcium $(0-500 \mu \mathrm{g} / \mathrm{mL})$ and linearity checks were performed for each analysis.

\section{Lectin Stain}

Leaflets from fresh porcine heart valves or commercial BHVs were embedded in optimal cutting temperature material (OCT; Miles Laboratories, Elkhart, Ind), snap-frozen in isopentane and dry ice, and cut on a cryostat into $4-\mu \mathrm{m}$-thick sections. Sections were processed by using standard methods for histochemical staining. The $\alpha$-Gal-specific lectin Griffonia simplicifolia-IB4 (GSIB4) requires divalent cations for binding. GSIB4 conjugated to horseradish peroxidase (EY Laboratories, San Mateo, Calif) was diluted to $2 \mu \mathrm{g} / \mathrm{mL}$ in Dulbecco's PBS (with $\mathrm{Ca}^{2+}$ and $\mathrm{Mn}^{2+}$ ) with $1 \%$ bovine serum albumin and incubated with the sections for 2 hours at $4{ }^{\circ} \mathrm{C}$. Lectin binding was visualized with 3,3'-diaminobenzidine staining (Sigma-Aldrich Corp, St Louis, Mo).

Paraffin-embedded formalin-fixed tissue sections were deparaffinized with 2 exchanges of Histo-Clear; rehydrated through a 100\%, 95\%, and $80 \%$ ethanol series; and rinsed in distilled water. Antigen retrieval was performed by immersing slides in citrate buffer $(10 \mathrm{mmol} / \mathrm{L}$ sodium citrate and $0.05 \%$ Tween $20, \mathrm{pH} 6.0$ ) at $95^{\circ} \mathrm{C}$ to $100^{\circ} \mathrm{C}$ for 20 minutes and allowed to cool to room temperature. Sections were rinsed in PBS and $0.05 \%$ Tween 20 and blocked with PBS and 1\% bovine serum albumin. Fluorescein isothiocyanate-conjugated GSIB4 was diluted as indicated above, and sections were stained at $4^{\circ} \mathrm{C}$ for 2 hours, washed with PBS and $0.05 \%$ Tween 20 , and examined with a Zeiss LSM51 confocal microscope. The specificity of lectin staining was determined by competition with $10 \mathrm{mmol} / \mathrm{L}$ free $\alpha$-Gal trisaccharide before staining.

\section{Pericardial Treatment and Implants}

Pericardium from wild-type and GTKO pigs was fixed and stored in $0.6 \%$ glutaraldehyde. After cutting into $0.8-\mathrm{cm}$ disks, the pericardium was washed in sterile saline overnight before antibody staining. All disks were incubated with affinity-purified human anti-Gal antibody (IgM and IgG) at $4 \mu \mathrm{g} / \mathrm{mL}$ in PBS for 1 hour at $4^{\circ} \mathrm{C}$ with gentle mixing. The density of disks for antibody labeling was 2 disks per milliliter. Disks were washed in an excess of sterile saline for 1 hour before implantation. After implantation, unused disks were labeled with goat anti-human IgG and IgM to document the level of antibody binding at the time of implantation.

\section{Rat and Rabbit Implants}

Twelve-day-old Wistar rats received subcutaneous implants of glutaraldehyde-fixed disks with and without prelabeling with anti-Gal antibody. Briefly, each recipient was initially anesthetized with ketamine and xylazine and maintained on $1 \%$ isoflurane. The skin was cleaned with $70 \%$ alcohol and Betadine, and 4 subcutaneous incisions were made along the dorsal surface, 2 per side. Disks were implanted smoothly against the body musculature, and the incisions were closed with sutures. Rabbit implantations were performed on $1.5-\mathrm{kg}$ rabbits. The hair was shaved from the dorsal surface, and the skin washed with Betadine. Five subcutaneous incisions were made per side, disks were implanted against the body wall, and the incisions were closed with sutures. In each instance a minimum of 10 (rats) or 40 (rabbits) disks were implanted and recovered for calcium determination. All animals were housed and received humane care in accordance with the standards established by the Institutional Animal Care and Use Committee of the Mayo Clinic and Foundation and as described in the "Guide for the care and use of laboratory animals" prepared by the Institute of Laboratory Animal Resources and published by the National Institutes of Health (publication no. 86-23, revised 1996).

\section{Statistical Analysis}

All data are presented as means \pm standard errors of the mean. Comparisons between experimental groups were tested for significance with the Student's $t$ test.

\section{RESULTS}

\section{Expression of $\alpha$-Gal in Porcine Tissues}

Expression of the $\alpha$-Gal antigen was detected by using fluorescein isothiocyanate- or horseradish peroxidase-conjugated GSIB4 lectin. High levels of lectin binding were detected in the matrix components of fresh wild-type porcine 

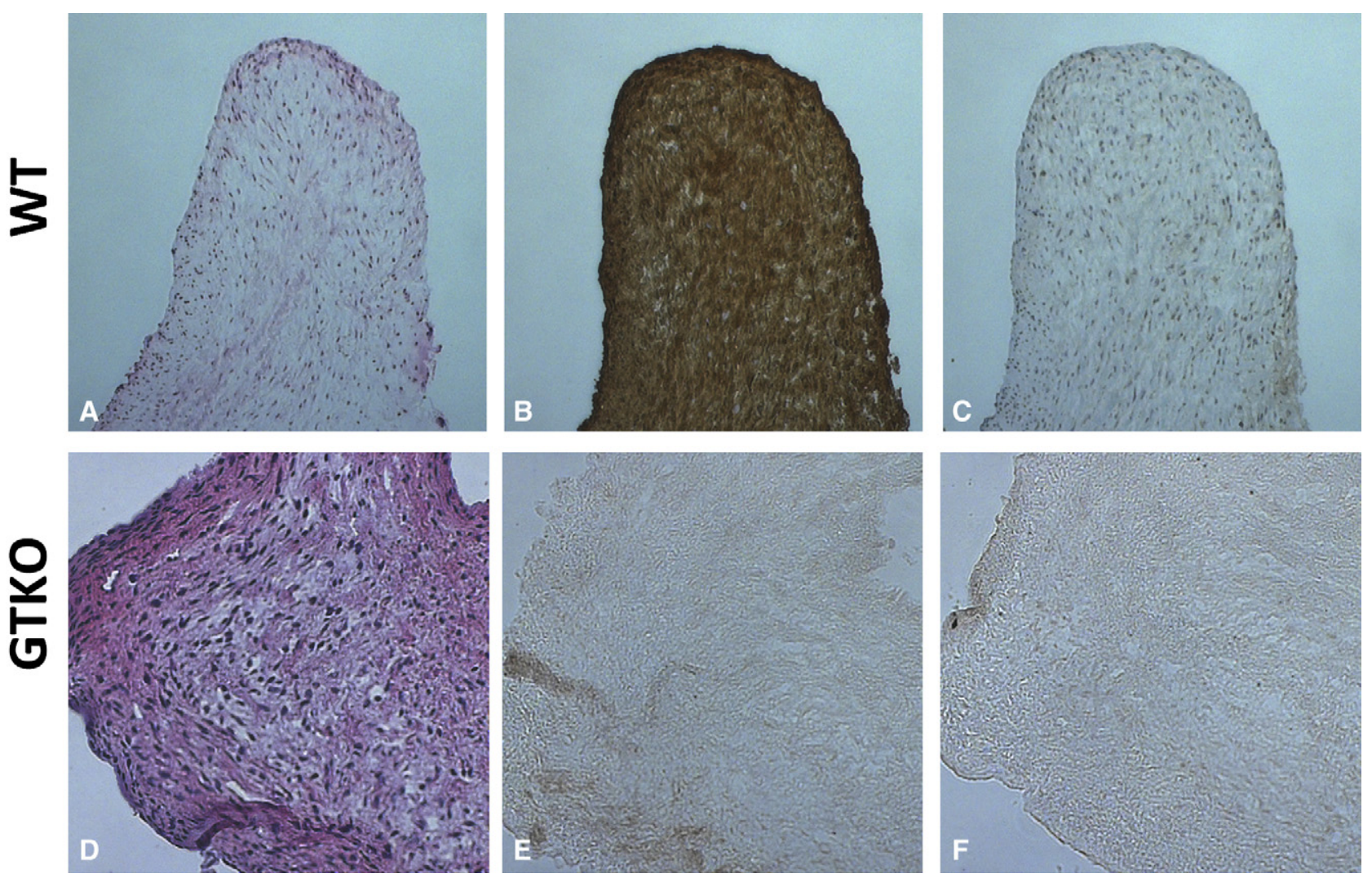

FIGURE 1. Comparison of GSIB4 staining of wild-type (WT; A-C) and GTKO (D-F) cardiac valves. A and D, Hematoxylin and eosin staining. B and E, Staining with Griffonia simplicifolia-IB4 (GSIB4)-horseradish peroxidase (HRP). C and F, GSIB4-HRP staining in the presence of $10 \mathrm{mmol} / \mathrm{L} \alpha$-Gal trisaccharide. Under these conditions, there is minimal staining because soluble $\alpha$-Gal blocks lectin binding to the tissue.

heart valves (Figure 1, $A$ and $B$ ). Staining appeared uniform across the valve and was specific for the $\alpha$-Gal carbohydrate because lectin binding was largely eliminated when the $\alpha$ Gal binding sites of GSIB4 were blocked by competition with soluble $\alpha$-Gal trisaccharide (Figure 1,C). In contrast, no specific staining was detected on fresh porcine heart valves from GTKO pigs (Figure $1, D-F$ ). The $\alpha$-Gal antigen is also expressed in the matrix of other tissues that might be used for the development of bioprosthetic devices. Intense staining of formalin-fixed porcine ligaments (Figure 2, A and $B$ ), tendons (Figure 2, $C$ and $D$ ), and pericardia (Figure $2, E$ and $F$ ) was evident in wild-type tissues. Staining was $\alpha$-Gal specific because competition with soluble $\alpha$-Gal trisaccharide effectively blocked GSIB4 binding (Figure 2, $B, D$, and $E$ inserts). Tissues from GTKO pigs did not exhibit appreciable GSIB4 binding (Figure 2, $H, J$, and $L$ ). After extensive fixation in $0.6 \%$ glutaraldehyde, the $\alpha$-Gal antigen remains accessible, as evidenced by lectin binding to wild-type but not GTKO porcine pericardium (Figure 3).

\section{$\alpha$-Gal Expression in Commercial Devices}

Bovine- and porcine-derived clinical BHVs were specifically stained with GSIB4 (Figure 4). The lectin staining indicated expression of $\alpha$-Gal was broadly distributed across the BHV matrix and was similar to that observed in fresh and glutaraldehyde-fixed porcine valves.

\section{Anti-Gal Antibody Accelerates Calcification}

To determine whether preformed anti-Gal antibody present in BHV recipients affected calcification of glutaraldehyde-fixed pericardium, we labeled wild-type and GTKO fixed pericardium with affinity-purified human anti-Gal antibody before implantation into juvenile rats or rabbits. Disks for implantation were processed as a batch, and after implantation, the remaining disks were stained to document the extent of antibody binding at the time of implantation. Wild-type fixed pericardium bound anti-Gal $\operatorname{IgM}$ and $\operatorname{IgG}$ and exhibited strong peroxidase staining (Figure 5, A). Little or no staining was evident on fixed GTKO pericardium. Wild-type disks coated with antibody and implanted into 12-day-old Wistar rats for 20 days showed extensive calcification on explants (Figure 5, B). Significantly increased calcification was evident in wildtype tissue incubated with anti-Gal antibody compared with that seen in the same tissue without antibody or compared with GTKO tissue with or without antibody. Calcification of GTKO samples was not affected by incubation with 

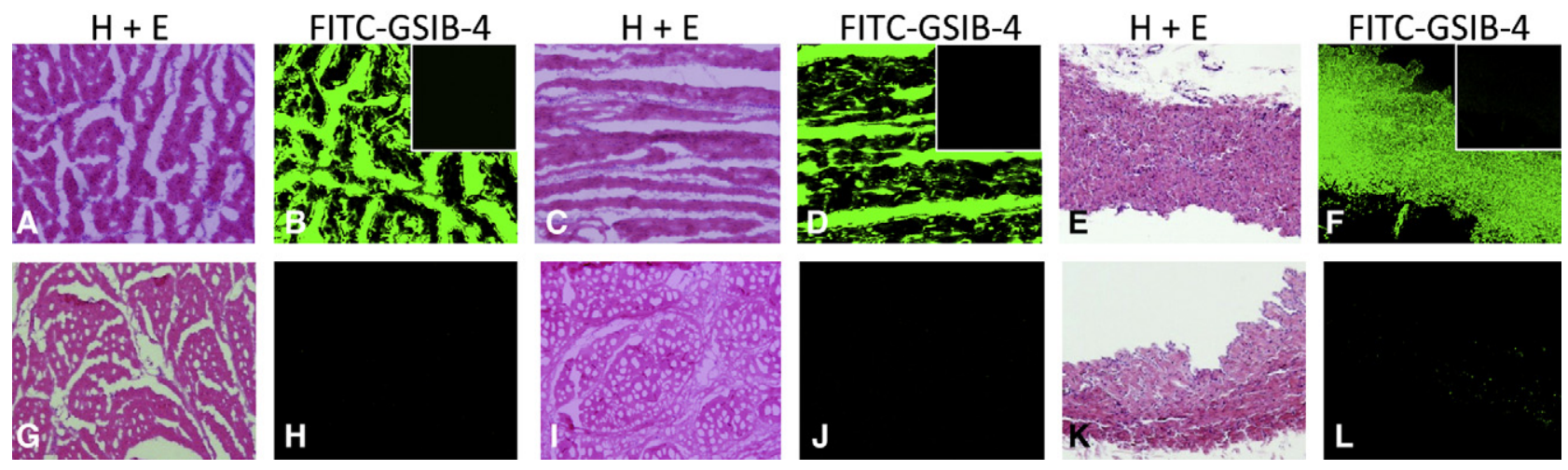

FIGURE 2. Comparison of wild-type and GTKO Gal expression. A-F, Wild-type porcine tissue. G-L, GTKO porcine tissue. The figure shows hematoxylin and eosin $(H+E)$ and fluorescein isothiocyanate-Griffonia simplicifolia-IB4 (FITC-GSIB4) staining of porcine ligaments (A and G), tendons (C and I), and pericardia (E and $\mathrm{K}$ ). The inserts in B, D, and F show wild-type FITC-GSIB4 staining in the presence of $\alpha$-Gal trisaccharide.

anti-Gal antibody and tended to be less than seen in wildtype samples under all conditions in the rat model. The same experiment was also performed with subcutaneous implants in rabbits (Figure 5,C). There was significantly greater calcification of wild-type glutaraldehyde-fixed porcine pericardium prelabeled with anti-Gal antibody compared with wild-type pericardium without antibody or GTKO pericardium with or without antibody.
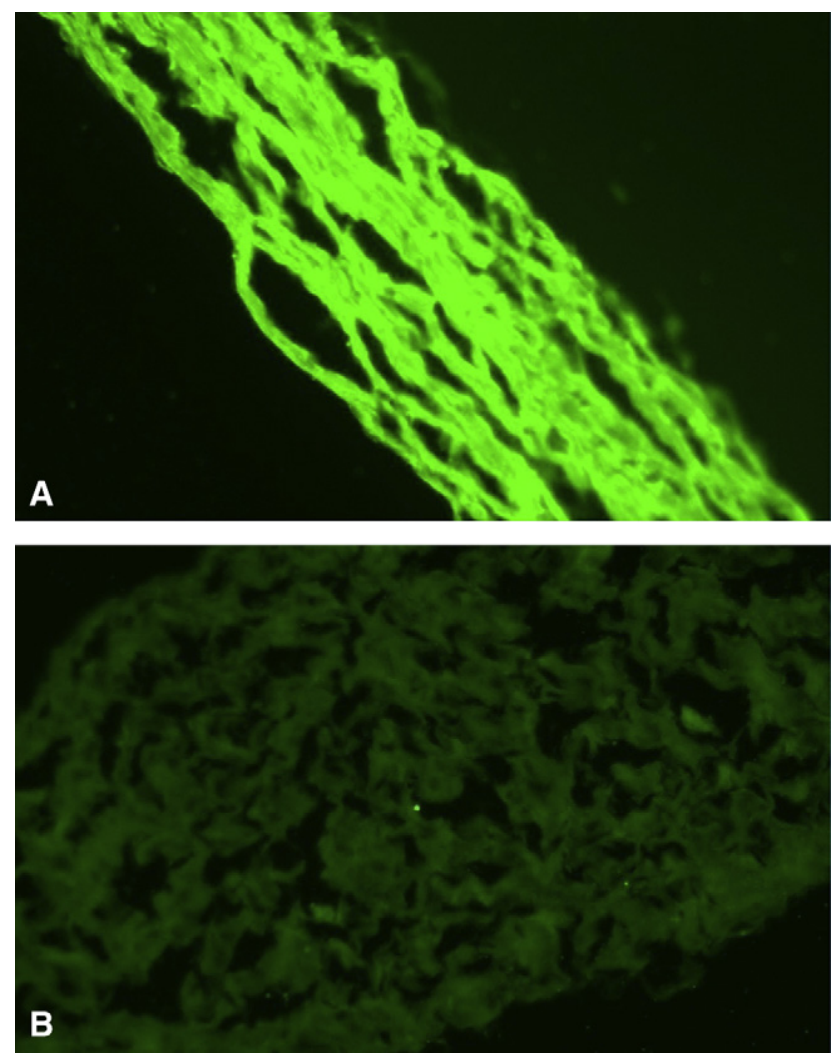

FIGURE 3. The Gal antigen is present after glutaraldehyde fixation in wild-type but not GTKO pericardium. Fluorescein isothiocyanate-Griffonia simplicifolia-IB4 staining of $0.6 \%$ glutaraldehyde-fixed wild-type (A) or GTKO (B) pericardium is shown.
Calcification of the explanted pericardium, as judged based on results of von Kossa staining, was largely confined to the pericardial disc and was generally not present in encapsulating cells. There was little evidence of cellular infiltration of the pericardium (Figure 5, $D-F$ ).

\section{DISCUSSION}

BHVs derived from animal tissues have been clinically used for more than 50 years. They are the preferred implants for older patients because they have a low level of thrombogenicity and generally do not require anticoagulation. In younger patients, however, BHVs exhibit a more rapid rate of degeneration. This degenerative process is not fully understood but is a multifaceted process including an induced immunologic response to the implant. BHVs as a component of left ventricular assist devices (LVADs) have been temporarily implanted into younger patient populations and might offer unique insight into the early immunologic response. Khan and colleagues, ${ }^{15}$ showed, in the absence of valve calcification, host tissue overgrowth, or valve endocarditis, very early binding and progressive infiltration of $\mathrm{CD} 8^{+}$mononuclear cells mainly on the nonflow sinus surface of BHVs recovered from implanted LVADs. This macrophage adhesion was detected in BHVs recovered after only 7 days of implantation, increased over time, and was associated with a progressive development of thrombus formation and cystic spaces in all valves. These changes were consistent with other studies ${ }^{16}$ that detected macrophage binding to the nonflow surface and IgG and complement deposition within the matrix after 37 days. These early inflammatory changes could result secondarily from tearing caused by mechanical or hemodynamic forces. Alternatively, Fc receptor-mediated antibody binding of macrophages might initiate an inflammatory response, which leads to collagen degradation, structural weakening, and leaflet tearing. We note that the immunologic response of LVAD recipients might not be identical to that of older patients with implanted bioprosthetic valves. These 


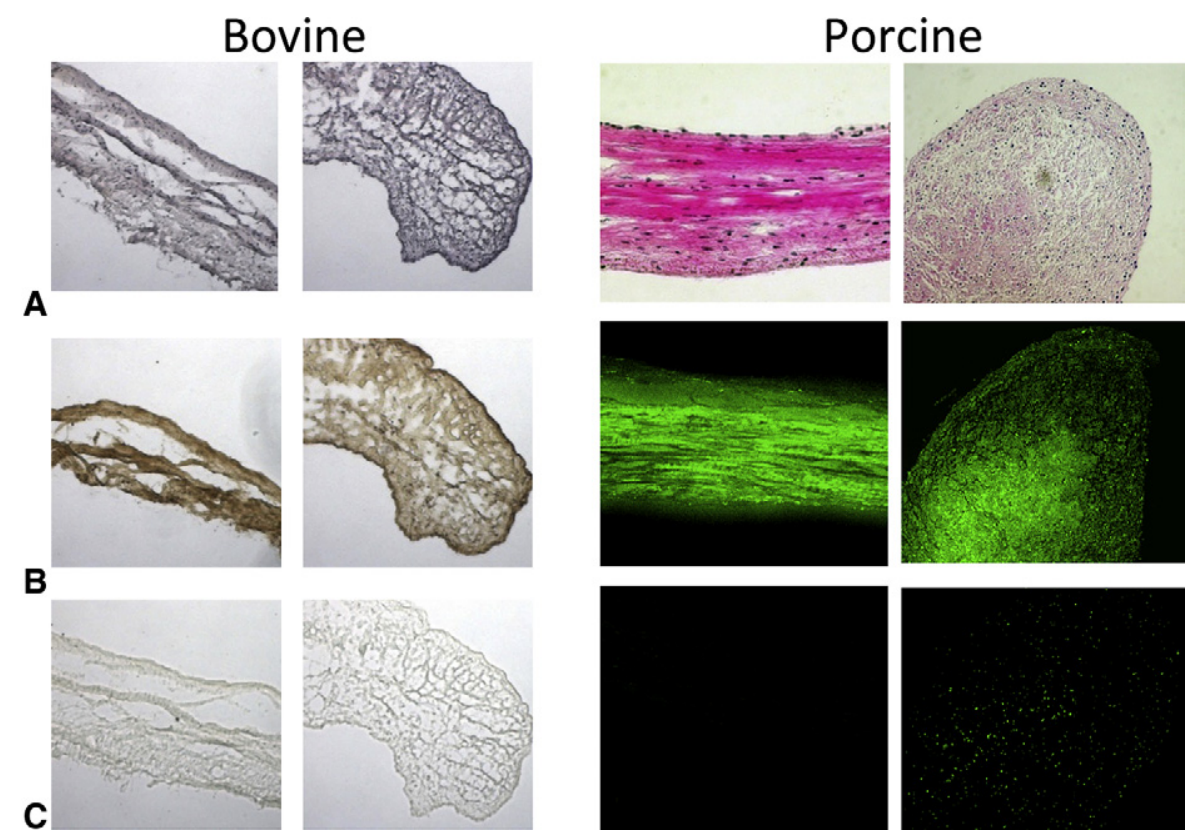

FIGURE 4. Griffonia simplicifolia-IB4 (GSIB4) staining of commercial bovine and porcine bioprosthetic valve devices. A, Hematoxylin and eosin staining. B, Staining of bovine devices with GSIB4-horseradish peroxidase (HRP) and of porcine devices with GSIB4-fluorescein isothiocyanate (FITC). C, Staining of bovine devices with GSIB4-HRP and of porcine devices with GSIB4-FITC in the presence of $10 \mathrm{mmol} / \mathrm{L}$ soluble $\alpha$-Gal trisaccharide. Under these conditions, lectin binding to the $\alpha$-Gal carbohydrate is effectively blocked.

differences might accelerate the inflammatory response in LVAD recipients. Still, antibody binding to BHVs and the potential for antibody-directed inflammatory reactions in patients with BHVs remains a possibility. In that respect it is important to note that an induced anti-Gal antibody response has been observed in BHV recipients. ${ }^{13}$

In this report we show that a well-characterized and universal human preformed antibody specificity, anti-Gal, is sufficient to accelerate calcification of glutaraldehydefixed $\alpha$-Gal-positive pericardium in both rat and rabbit subcutaneous implant models. We further show that glutaraldehyde-fixed pericardium from GTKO pigs does not bind anti-Gal antibody and shows significantly lower levels of calcification in these models. Our results are a significant extension of previous work that demonstrated that disks incubated with graft-specific antibody exhibited accelerated calcification ${ }^{11}$ because in our case anti-Gal antibody is preformed and requires no immunization. Although addition of antibody directly to the pericardium might overestimate its effects on calcification, our results indicate the potential for preformed antibody-directed inflammation as an immunologic mechanism to initiate calcification. The rat subcutaneous implant model is an inexpensive, reliable preclinical testing method used to assess the effect of new manufacturing techniques to prevent calcification. Processing techniques that reduce calcification in this model have led to improved commercial BHVs, but the results of this model do not always reflect the outcomes in patients. Functional testing of new BHVs with full blood exposure is generally performed in juvenile sheep, in which BHV calcification can be observed within 3 to 6 months. ${ }^{17,18}$ Sheep produce the $\alpha$-Gal antigen, however, and therefore the affects of the anti-Gal antibody cannot be determined in this model. Further examination of the role of anti-Gal antibody in BHV calcification with full exposure to blood could be performed by using implantation into Gal-deficient pigs. The slow rate of calcification, expense, and ethical considerations generally preclude testing new BHVs in nonhuman primates.

This study cannot directly link the affects of anti-Gal antibody in the subcutaneous model to macrophage adhesion and valve degeneration in patients with LVADs, but it does confirm previous reports that device-specific antibody can increase calcification ${ }^{11}$ and importantly demonstrates that this can occur with preformed antibody specificity and does not necessarily require sensitization. The historic experience with the rat subcutaneous model and our results suggest that processes or materials which limit antibody binding to BHVs will help to prevent calcification.

Expression of the $\alpha$-Gal antigen in GTKO pigs has been characterized in a variety of tissues, with emphasis on vascular organs. We show, using GSIB4 staining, a high level of $\alpha$-Gal expression in the matrix of the pericardia, ligaments, and tendons of wild-type tissues and an absence of expression in corresponding GTKO tissues. These observations are consistent with the results of Stone and coworkers, ${ }^{4}$ who used a competitive enzyme-linked immunosorbent assay to show the presence of up to $10^{11} \mathrm{Gal}$ 


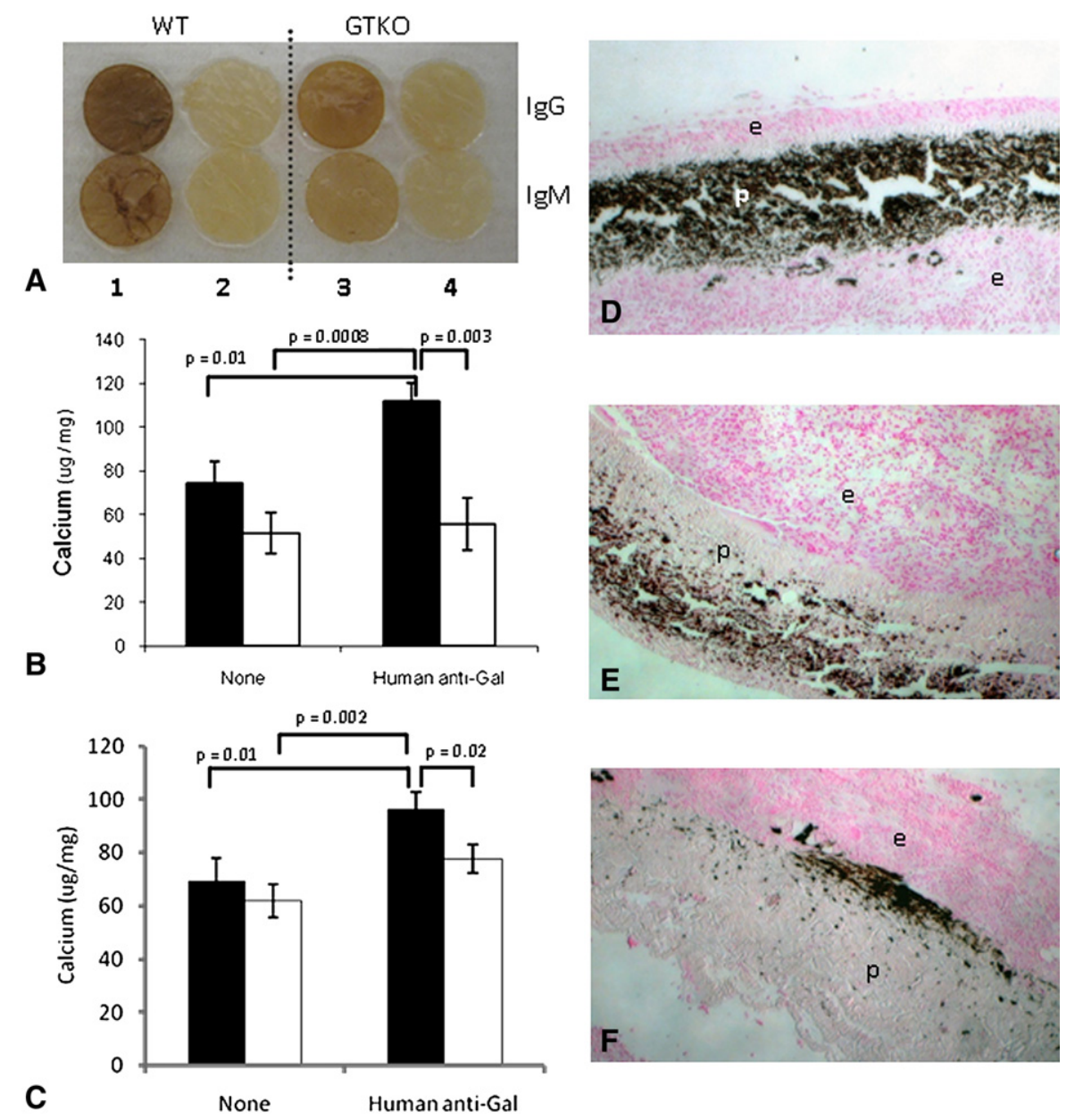

FIGURE 5. Implantation and calcification of glutaraldehyde-fixed porcine pericardium with and without anti-Gal antibody binding. A, Whole pericardial disks stained at the time of implantation with goat anti-human immunoglobulin $(\mathrm{Ig}) \mathrm{G}$ and $\mathrm{IgM}$ to detect the binding of anti-Gal antibody. Lanes 1 and 3 show specific staining for $\operatorname{IgG}$ (top) and $\operatorname{IgM}$ (bottom) on wild-type (WT) and GTKO disks, respectively. Lanes 2 and 4 show background binding with only the goat anti-human IgG (top) or IgM (bottom). B, Calcification of wild-type (black bars) and GTKO (white bars) pericardium 20 days after subcutaneous implantation into 12-day-old rats. C, Calcification of wild-type (black bars) and GTKO (white bars) pericardium 20 days after subcutaneous implantation into 1.5-kg rabbits. Pericardial disks were implanted without exposure to human anti-Gal antibody (None) or after incubation with human anti-Gal antibody (Human anti-Gal). Error bars represent the standard error of the mean. D and F, Representative von Kossa staining of explanted pericardium. D, Wildtype disc preincubated with anti-Gal antibody before implantation. E, Wild-type disc without anti-Gal antibody before implantation. F, GTKO disc without anti-Gal antibody before implantation. Von Kossa staining of calcium (black) is largely confined to the pericardium $(p)$ and is absent in the eosin-stained encapsulating cells $(e)$.

epitopes per milligram of porcine tendon. We have investigated the antigenicity of GTKO endothelial cell antigens after cardiac xenotransplantation. ${ }^{19}$ Although we cannot exclude that additional carbohydrate antigen might be detected by non-Gal antibodies in human subjects and nonhuman primates, it appears that the majority of non-Gal antibodies are directed to common porcine proteins. The antigenicity of these protein antigens is expected to be strongly affected by glutaraldehyde cross-linking. We also note that the $\alpha$-Gal antigen has been used to augment the effectiveness of vaccines in which it enhances immune responses by increasing the formation and transport of antigen complexes to lymph nodes for processing by antigen-presenting cells. ${ }^{20}$ In this context the elimination of this antigen might further reduce the antigenicity of BHVs. Our observation of enhanced calcification when anti-Gal antibody is bound to fixed pericardium suggests that the absence of this antigen in tissue relevant for engineering bioprosthetic devices is advantageous and that materials from GTKO pigs might make a significant contribution to the development of improved bioprosthetic devices, including BHVs.

\section{References}

1. McGregor CG, Davies WR, Oi K, et al. Cardiac xenotransplantation: recent preclinical progress with 3-month median survival. J Thorac Cardiovasc Surg. 2005;130:844-51. 
2. Cooper DKC, Good AH, Koren E, et al. Identification of alpha-galactosyl and other carbohydrate epitopes that are bound by human anti-pig antibodies: relevance to discordant xenografting in man. Transpl Immunol. 1993;1: 198-205.

3. Galili U, Shohet SB, Kobrin E, Stults CM, Macher BA. Man, apes, and old world monkeys differ from other mammals in the expression of alpha-Galactosyl epitopes on nucleated cells. J Biol Chem. 1988;263:17755-62.

4. Stone KR, Abdel-Motal UM, Walgenbach AW, Turek TJ, Galili U. Replacement of human anterior cruciate ligaments with pig ligaments: a model for anti-nongal antibody response in long-term xenotransplantation. Transplantation. 2007;83:211-9.

5. Doenz U, Nydegger UE, Kueng A, Carrel T, Mohacsi P. Anti-Galalpha1-3Gal $\mathrm{IgM} / \mathrm{IgG}$ antibody levels in infants: do they have a clinical relevance in pediatric xenotransplantation? J Heart Lung Transplant. 2000;19:1108-13.

6. Kozlowski T, Ierino FL, Lambrigts D, et al. Depletion of anti-Gala1-3Gal antibody in baboons by specific a-Gal immunoaffinity columns. Xenotransplantation. 1998;5:122-31.

7. Byrne GW, Schwarz A, Fesi JR, et al. Evaluation of different alpha-Galactosyl glycoconjugates for use in xenotransplantation. Bioconjug Chem. 2002;13: 571-81.

8. Lai L, Kolber-Simonds D, Park K-W, et al. Production of a-1,3-galactosyltransferase knockout pigs by nuclear transfer cloning. Science. 2002;295:1089-92.

9. Sharma A, Naziruddin B, Cui C, et al. Pig cells that lack the gene for alpha1-3 galactosyltransferase express low levels of the gal antigen. Transplantation. 2003; $75: 430-6$

10. Kuwaki K, Tseng YL, Dor FJ, et al. Heart transplantation in baboons using alpha1,3-galactosyltransferase gene-knockout pigs as donors: initial experience. Nat Med. 2005;11:29-31.

11. Human P, Zilla P. Characterization of the immune response to valve bioprostheses and its role in primary tissue failure. Ann Thorac Surg. 2001;71(suppl): S385-8.

12. Manji RA, Zhu LF, Nijjar NK, et al. Glutaraldehyde-fixed bioprosthetic heart valve conduits calcify and fail from xenograft rejection. Circulation. 2006;114: 318-27.

13. Konakci KZ, Bohle B, Blumer R, et al. Alpha-Gal on bioprostheses: xenograft immune response in cardiac surgery. Eur J Clin Invest. 2005;35:17-23.

14. Lin SS, Hanaway MJ, Gonzalez-Stawinski GV, et al. The role of anti-Gala1-3Gal antibodies in acute vascular rejection and accommodation of xenografts. Transplantation. 2000;70:1667-74

15. Khan NA, Butany J, Zhou T, Ross HJ, Rao V. Pathological findings in explanted prosthetic heart valves from ventricular assist devices. Pathology. 2008;40: 377-84.

16. Moczar M, Houel R, Ginat M, Clerin V, Wheeldon D, Loisance D. Structural changes in porcine bioprosthetic valves of a left ventricular assist system in human patients. J Heart Valve Dis. 2000;9:88-96.

17. Carpentier A, Nashef A, Carpentier S, Ahmed A, Goussef N. Techniques for prevention of calcification of valvular bioprostheses. Circulation. 1984;70(Suppl I): I165-8.

18. Carpentier SM, Chen L, Shen M, et al. Heat treatment mitigates calcification of valvular bioprostheses. Ann Thorac Surg. 1998;66(Suppl):S264-6.

19. Byrne GW, Stalboerger PG, Davila E, et al. Proteomic identification of non-Gal antibody targets after pig-to-primate cardiac xenotransplantation. Xenotransplantation. 2008; 15:268-76.

20. Abdel-Motal UM, Wigglesworth K, Galili U. Mechanism for increased immunogenicity of vaccines that form in vivo immune complexes with the natural anti-Gal antibody. Vaccine. 2009;27:3072-82.

\section{Discussion}

Dr R. Duane Davis (Durham, NC). Chris, my congratulations. First of all, let me just put it in perspective. You are demonstrating that it is sufficient to have anti-Gal to cause this injury and calci- fication, and therefore it does really raise the following question: Can we have a better biologic valve? That is incredibly important.

You did game the system a little bit. We know that there are a lot of non-Gal anti-swine or other antibodies present, and you did not use those in this experiment. That has a lot of variability. It can be as little as $5 \%$ of the anti-swine antibody, or it can be as high as $35 \%$. What happens when you use those antibodies in this model?

Dr McGregor. Clearly there are multiple non-Gal antibodies, as you say, of variable quantities, somewhere up to $20 \%$. We started by dealing with the major antibody, which was $80 \%$ of the bulk. We had to start somewhere. Do non-Gal antibodies matter? I am sure they do, but I do not think that we have sufficiently defined their specificity as of this time. The major effort in our laboratory now is to identify and specify those non-Gal antibodies so that we can eliminate them by further genetic alteration of the pig. They are important, but they are probably at the $20 \%$ level, as you say.

Dr Davis. Let me kind of follow this up in terms of-you have this model, which obviously it is not putting it into the blood. Is there a way that we can actually test this in vivo in an animal model, or do we just need to go to human applications?

Dr McGregor. That is a wonderful and very important question. The animal models are all flawed, and we have known it for a long time. The rat model is simply the best we have. Over 40 years it has correlated not unreasonably with subsequent clinical performance in terms of improvement of processing. The sheep model, because of the Gal issue, of course, is not applicable.

We currently have a number of nonhuman primates with Galpositive and Gal-negative biological mitral valves in place. But this study takes an extraordinary length of time, and I am not convinced it is going to give us the answers because we might have to run them for up to 10 years. The preponderance of evidence now is so powerful that my own belief is that we should go to a clinical trial in young patients using these Gal knockout tissues because there is no downside and because the potential upside is so important that I think the evidence suggests we should go ahead at this time.

Dr Davis. I really could not agree with you more on that. The aspect now that you know that there is non-Gal antibody-and in this situation you do not need to necessarily have tissues that have ongoing expression per se-are there ways that you can manipulate these Gal knockout tissues to make them appear to function better because of the other immunologic potential injuries that they could have?

Dr McGregor. Clearly the amount of antigenic tissue in any heart valve is in part determined by the intensity and durability of pretreatment and also affected by the treatment that any recipient would receive. Therefore in several of these studies, for example, steroid therapy diminished the antigenicity. That is not something we would want to do in patients, but I think with processing, there are always going to be areas where we can improve in terms of residual antigenicity. The question is this: Will we pay a price chemically in terms of collagen cross-linking and so forth 